\title{
A relation between two simple Hardy-Mulholland-type inequalities with parameters
}

Qiang Chen ${ }^{*}$, Yanping Shi ${ }^{2}$ and Bicheng Yang ${ }^{3}$

\section{"Correspondence:}

cq_c123@163.com

'Department of Computer Science,

Guangdong University of

Education, Guangzhou, Guangdong

51003, P.R. China

Full list of author information is

available at the end of the article

\begin{abstract}
By means of weight coefficients and the technique of real analysis, a new

Hardy-Mulholland-type inequality with the kernel

$$
K_{\lambda}(m, n):=\frac{1}{\ln ^{\lambda} U_{m}+\ln ^{\lambda} V_{n}+\alpha\left|\ln ^{\lambda} U_{m}-\ln ^{\lambda} V_{n}\right|}
$$

$(-1<\alpha \leq 1,0<\lambda \leq 2 ; m, n \in \mathbf{N} \backslash\{1\})$ and a best possible constant factor is provided, which is a relation between two simple Hardy-Mulholland-type inequalities with parameters. The equivalent forms, the operator expression with the norm, and some particular inequalities are studied. The lemmas and theorems of this paper provide an extensive account of this type of inequalities.
\end{abstract}

MSC: $26 \mathrm{D} 15 ; 47 \mathrm{~A} 07$

Keywords: Hardy-Mulholland-type inequality; weight coefficient; equivalent form; operator; norm

\section{Introduction}

Suppose that $p>1, \frac{1}{p}+\frac{1}{q}=1, a_{m}, b_{n} \geq 0, a=\left\{a_{m}\right\}_{m=1}^{\infty} \in l^{p}, b=\left\{b_{n}\right\}_{n=1}^{\infty} \in l^{q},\|a\|_{p}=$ $\left(\sum_{m=1}^{\infty} a_{m}^{p}\right)^{\frac{1}{p}}>0,\|b\|_{q}>0$. We have the following Hardy-Hilbert inequality:

$$
\sum_{n=1}^{\infty} \sum_{m=1}^{\infty} \frac{a_{m} b_{n}}{m+n}<\frac{\pi}{\sin \left(\frac{\pi}{p}\right)}\|a\|_{p}\|b\|_{q}
$$

where the constant factor $\frac{\pi}{\sin (\pi / p)}$ is the best possible ( $c f$. [1]). We still have the following Hilbert-type inequality:

$$
\sum_{n=1}^{\infty} \sum_{m=1}^{\infty} \frac{a_{m} b_{n}}{\max \{m, n\}}<p q\|a\|_{p}\|b\|_{q}
$$

with the best possible constant factor $p q$ ( $c f$. [1]). Also the following Mulholland inequality was given with the best possible constant factor $\frac{\pi}{\sin (\pi / p)}\left(c f .[1]\right.$, Theorem 343 , replacing $\frac{a_{m}}{m}$, 
$\frac{b_{n}}{n}$ by $\left.a_{m}, b_{n}\right)$ :

$$
\sum_{n=2}^{\infty} \sum_{m=2}^{\infty} \frac{a_{m} b_{n}}{\ln m n}<\frac{\pi}{\sin \left(\frac{\pi}{p}\right)}\left(\sum_{m=2}^{\infty} \frac{a_{m}^{p}}{m^{1-p}}\right)^{\frac{1}{p}}\left(\sum_{n=2}^{\infty} \frac{b_{n}^{q}}{n^{1-q}}\right)^{\frac{1}{q}} .
$$

Inequalities (1)-(3) are important in analysis and its applications (cf. [1-5]).

If $\mu_{i}, v_{j}>0(i, j \in \mathbf{N}=\{1,2, \ldots\})$,

$$
U_{m}:=\sum_{i=1}^{m} \mu_{i}, \quad V_{n}:=\sum_{j=1}^{n} v_{j} \quad(m, n \in \mathbf{N})
$$

then we have the following Hardy-Hilbert-type inequality ( $c f$. Theorem 321 of [1], replacing $\mu_{m}^{1 / q} a_{m}$ and $v_{n}^{1 / p} b_{n}$ by $a_{m}$ and $b_{n}$ ):

$$
\sum_{n=1}^{\infty} \sum_{m=1}^{\infty} \frac{a_{m} b_{n}}{U_{m}+V_{n}}<\frac{\pi}{\sin \left(\frac{\pi}{p}\right)}\left(\sum_{m=1}^{\infty} \frac{a_{m}^{p}}{\mu_{m}^{p-1}}\right)^{\frac{1}{p}}\left(\sum_{n=1}^{\infty} \frac{b_{n}^{q}}{v_{n}^{q-1}}\right)^{\frac{1}{q}} .
$$

For $\mu_{i}=v_{j}=1(i, j \in \mathbf{N})$, inequality (5) reduces to (1).

By introducing an independent parameter $\lambda \in(0,1]$, in 1998, Yang [6] gave an extension of the integral analogous of (1) with the kernel $\frac{1}{(x+y)^{\lambda}}$ for $p=q=2$. Following [6], Yang [7] gave extensions of (1) and (2) as follows.

If $\lambda_{1}, \lambda_{2} \in \mathbf{R}, \lambda_{1}+\lambda_{2}=\lambda, k_{\lambda}(x, y)$ is a finite non-negative homogeneous function of degree $-\lambda$, with

$$
k\left(\lambda_{1}\right)=\int_{0}^{\infty} k_{\lambda}(t, 1) t^{\lambda_{1}-1} d t \in \mathbf{R}_{+}
$$

and $k_{\lambda}(x, y) x^{\lambda_{1}-1}\left(k_{\lambda}(x, y) y^{\lambda_{2}-1}\right)$ is decreasing with respect to $x>0(y>0), \phi(x)=x^{p\left(1-\lambda_{1}\right)-1}$, $\psi(x)=x^{q\left(1-\lambda_{2}\right)-1}$, then for $a_{m}, b_{n} \geq 0$,

$$
a=\left\{a_{m}\right\}_{m=1}^{\infty} \in l_{p, \phi}=\left\{a ;\|a\|_{p, \phi}:=\left(\sum_{m=1}^{\infty} \phi(m)\left|a_{m}\right|^{p}\right)^{\frac{1}{p}}<\infty\right\},
$$

$b=\left\{b_{n}\right\}_{n=1}^{\infty} \in l_{q, \psi},\|a\|_{p, \phi},\|b\|_{q, \psi}>0$, we have

$$
\sum_{n=1}^{\infty} \sum_{m=1}^{\infty} k_{\lambda}(m, n) a_{m} b_{n}<k\left(\lambda_{1}\right)\|a\|_{p, \phi}\|b\|_{q, \psi}
$$

where the constant factor $k\left(\lambda_{1}\right)$ is still the best possible. Clearly, for $\lambda=1, \lambda_{1}=\frac{1}{q}, \lambda_{2}=\frac{1}{p}$, $k_{1}(x, y)=\frac{1}{x+y}\left(\frac{1}{\max \{x, y\}}\right)$, inequality $(6)$ reduces to $(1)((2))$.

Some other new results including multidimensional Hilbert-type inequalities, HardyHilbert-type inequalities and Hardy-Mulholland-type inequalities are provided by [8-30].

In this paper, by means of weight coefficients and the technique of real analysis, a new Hardy-Mulholland-type inequality with the following kernel:

$$
K_{\lambda}(m, n):=\frac{1}{\ln ^{\lambda} U_{m}+\ln ^{\lambda} V_{n}+\alpha\left|\ln ^{\lambda} U_{m}-\ln ^{\lambda} V_{n}\right|}
$$


$(-1<\alpha \leq 1,0<\lambda \leq 2 ; m, n \in \mathbf{N} \backslash\{1\})$ and a best possible constant factor is provided, which is a relation between two simple Hardy-Mulholland-type inequalities similarly to (2) and (3). The equivalent forms, the operator expressions with the norm and some particular inequalities are studied. The lemmas and theorems of this paper provide an extensive account of this type of inequalities.

\section{An example and some lemmas}

Example 1 For $-1<\alpha \leq 1,0<\lambda_{1}, \lambda_{2} \leq 1, \lambda_{1}+\lambda_{2}=\lambda$, we set

$$
k_{\lambda}(x, y):=\frac{1}{x^{\lambda}+y^{\lambda}+\alpha\left|x^{\lambda}-y^{\lambda}\right|} \quad\left((x, y) \in \mathbf{R}_{+}^{2}=\mathbf{R}_{+} \times \mathbf{R}_{+}\right)
$$

Then by (7), it follows that $K_{\lambda}(m, n)=k_{\lambda}\left(\ln U_{m}, \ln V_{n}\right)$. We find for $-1<\alpha \leq 1, \lambda_{1}, \lambda_{2}>0$,

$$
\begin{aligned}
0 & <k_{\alpha}\left(\lambda_{1}\right):=\int_{0}^{\infty} k_{\lambda}(1, t) t^{\lambda_{2}-1} d t=\int_{0}^{\infty} k_{\lambda}(t, 1) t^{\lambda_{1}-1} d t \\
& =\int_{0}^{\infty} \frac{t^{\lambda_{1}-1} d t}{t^{\lambda}+1+\alpha\left|t^{\lambda}-1\right|}=\int_{0}^{1} \frac{t^{\lambda_{1}-1}+t^{\lambda_{2}-1}}{1+\alpha+(1-\alpha) t^{\lambda}} d t \\
& \leq \int_{0}^{1} \frac{t^{\lambda_{1}-1}+t^{\lambda_{2}-1}}{1+\alpha} d t=\frac{1}{1+\alpha}\left(\frac{1}{\lambda_{1}}+\frac{1}{\lambda_{2}}\right)<\infty,
\end{aligned}
$$

namely, $k_{\alpha}\left(\lambda_{1}\right) \in \mathbf{R}_{+}$.

(1) In the following, we express $k_{\alpha}\left(\lambda_{1}\right)$ in a few cases.

(i) For $\alpha=0$, we obtain

$$
\begin{aligned}
k_{0}\left(\lambda_{1}\right) & =\int_{0}^{\infty} \frac{t^{\lambda_{1}-1}}{t^{\lambda}+1} d t \\
& =\frac{1}{\lambda} \int_{0}^{\infty} \frac{u^{\left(\lambda_{1} / \lambda\right)-1}}{u+1} d u=\frac{\pi}{\lambda \sin \left(\frac{\pi \lambda_{1}}{\lambda}\right)}
\end{aligned}
$$

(ii) For $\alpha=1$, we obtain

$$
\begin{aligned}
k_{1}\left(\lambda_{1}\right) & =\int_{0}^{\infty} \frac{t^{\lambda_{1}-1}}{t^{\lambda}+1+\left|t^{\lambda}-1\right|} d t=\int_{0}^{\infty} \frac{t^{\lambda_{1}-1}}{2 \max \left\{t^{\lambda}, 1\right\}} d t \\
& =\frac{1}{2} \int_{0}^{1}\left(t^{\lambda_{1}-1}+t^{\lambda_{2}-1}\right) d t=\frac{\lambda}{2 \lambda_{1} \lambda_{2}}
\end{aligned}
$$

(iii) For $0<\alpha<1,0<\frac{1-\alpha}{1+\alpha}<1$, in view of (9) and the Lebesgue term by term integration theorem $(c f .[31])$, we find

$$
\begin{aligned}
k_{\alpha}\left(\lambda_{1}\right) & =\frac{1}{1+\alpha} \int_{0}^{1} \frac{t^{\lambda_{1}-1}+t^{\lambda_{2}-1}}{1+\frac{1-\alpha}{1+\alpha} t^{\lambda}} d t \\
& =\frac{1}{1+\alpha} \int_{0}^{1}\left(t^{\lambda_{1}-1}+t^{\lambda_{2}-1}\right) \sum_{k=0}^{\infty}(-1)^{k}\left(\frac{1-\alpha}{1+\alpha}\right)^{k} t^{\lambda k} d t \\
& =\frac{1}{1+\alpha} \int_{0}^{1}\left(t^{\lambda_{1}-1}+t^{\lambda_{2}-1}\right) \sum_{j=0}^{\infty}\left(\frac{1-\alpha}{1+\alpha}\right)^{2 j}\left(1-\frac{1-\alpha}{1+\alpha} t^{\lambda}\right) t^{2 \lambda j} d t
\end{aligned}
$$




$$
\begin{aligned}
& =\frac{1}{1+\alpha} \sum_{j=0}^{\infty} \int_{0}^{1}\left(t^{\lambda_{1}-1}+t^{\lambda_{2}-1}\right)\left(\frac{1-\alpha}{1+\alpha}\right)^{2 j}\left(1-\frac{1-\alpha}{1+\alpha} t^{\lambda}\right) t^{2 \lambda j} d t \\
& =\frac{1}{1+\alpha} \sum_{k=0}^{\infty}(-1)^{k}\left(\frac{1-\alpha}{1+\alpha}\right)^{k} \int_{0}^{1}\left(t^{\lambda_{1}-1}+t^{\lambda_{2}-1}\right) t^{\lambda k} d t \\
& =\frac{1}{1+\alpha} \sum_{k=0}^{\infty}(-1)^{k}\left(\frac{1-\alpha}{1+\alpha}\right)^{k}\left(\frac{1}{\lambda k+\lambda_{1}}+\frac{1}{\lambda k+\lambda_{2}}\right) .
\end{aligned}
$$

(iv) For $-1<\alpha<0,0<\frac{1+\alpha}{1-\alpha}<1$, by (9) and the Lebesgue term by term integration theorem (cf. [31]), we find

$$
\begin{aligned}
& k_{\alpha}\left(\lambda_{1}\right)=\frac{1}{1+\alpha} \int_{0}^{1} \frac{t^{\lambda_{1}-1}+t^{\lambda_{2}-1}}{1+\frac{1-a}{1+a} t^{\lambda}} d t \\
& \stackrel{\nu=\frac{1+\alpha}{1-\alpha}}{=} \frac{1}{\lambda(1+\alpha)} \int_{\frac{1+\alpha}{1-\alpha}}^{\infty} \frac{1}{v+1} \\
& \times\left[\left(\frac{1+\alpha}{1-\alpha}\right)^{\frac{\lambda_{1}}{\lambda}} v^{\frac{-\lambda_{1}}{\lambda}}+\left(\frac{1+\alpha}{1-\alpha}\right)^{\frac{\lambda_{2}}{\lambda}} v^{\frac{-\lambda_{2}}{\lambda}}\right] d v \\
& =\frac{1}{\lambda(1+\alpha)}\left(\frac{1+\alpha}{1-\alpha}\right)^{\frac{\lambda_{1}}{\lambda}} \int_{0}^{\infty} \frac{1}{v+1} v^{\left(1-\frac{\lambda_{1}}{\lambda}\right)-1} d v \\
& +\frac{1}{\lambda(1+\alpha)}\left(\frac{1+\alpha}{1-\alpha}\right)^{\frac{\lambda_{2}}{\lambda}} \int_{0}^{\infty} \frac{1}{v+1} v^{\left(1-\frac{\lambda_{2}}{\lambda}\right)-1} d v \\
& -\frac{1}{\lambda(1+\alpha)} \int_{0}^{\frac{1+\alpha}{1-\alpha}} \frac{1}{v+1} \\
& \times\left[\left(\frac{1+\alpha}{1-\alpha}\right)^{\frac{\lambda_{1}}{\lambda}} v^{\frac{-\lambda_{1}}{\lambda}}+\left(\frac{1+\alpha}{1-\alpha}\right)^{\frac{\lambda_{2}}{\lambda}} v^{\frac{-\lambda_{2}}{\lambda}}\right] d v \\
& =\frac{1}{\lambda(1+\alpha)}\left(\frac{1+\alpha}{1-\alpha}\right)^{\frac{\lambda_{1}}{\lambda}} \frac{\pi}{\sin \left(\frac{\pi \lambda_{2}}{\lambda}\right)} \\
& +\frac{1}{\lambda(1+\alpha)}\left(\frac{1+\alpha}{1-\alpha}\right)^{\frac{\lambda_{2}}{\lambda}} \frac{\pi}{\sin \left(\frac{\pi \lambda_{1}}{\lambda}\right)} \\
& -\frac{1}{\lambda(1+\alpha)} \int_{0}^{\frac{1+\alpha}{1-\alpha}} \sum_{k=0}^{\infty}(-1)^{k} v^{k} \\
& \times\left[\left(\frac{1+\alpha}{1-\alpha}\right)^{\frac{\lambda_{1}}{\lambda}} v^{\frac{-\lambda_{1}}{\lambda}}+\left(\frac{1+\alpha}{1-\alpha}\right)^{\frac{\lambda_{2}}{\lambda}} v^{\frac{-\lambda_{2}}{\lambda}}\right] d v \\
& =\frac{1}{\lambda(1+\alpha)}\left[\left(\frac{1+\alpha}{1-\alpha}\right)^{\frac{\lambda_{1}}{\lambda}}+\left(\frac{1+\alpha}{1-\alpha}\right)^{\frac{\lambda_{2}}{\lambda}}\right] \frac{\pi}{\sin \left(\frac{\pi \lambda_{1}}{\lambda}\right)} \\
& -\frac{1}{\lambda(1+\alpha)} \int_{0}^{\frac{1+\alpha}{1-\alpha}} \sum_{k=0}^{\infty}(-1)^{k} v^{k} \\
& \times\left[\left(\frac{1+\alpha}{1-\alpha}\right)^{\frac{\lambda_{1}}{\lambda}} v^{\frac{-\lambda_{1}}{\lambda}}+\left(\frac{1+\alpha}{1-\alpha}\right)^{\frac{\lambda_{2}}{\lambda}} v^{\frac{-\lambda_{2}}{\lambda}}\right] d v
\end{aligned}
$$




$$
\begin{aligned}
& =\frac{1}{\lambda(1+\alpha)}\left[\left(\frac{1+\alpha}{1-\alpha}\right)^{\frac{\lambda_{1}}{\lambda}}+\left(\frac{1+\alpha}{1-\alpha}\right)^{\frac{\lambda_{2}}{\lambda}}\right] \frac{\pi}{\sin \left(\frac{\pi \lambda_{1}}{\lambda}\right)} \\
& -\frac{1}{\lambda(1+\alpha)} \int_{0}^{\frac{1+\alpha}{1-\alpha}} \sum_{k=0}^{\infty}\left(v^{2 k}-v^{2 k+1}\right) \\
& \times\left[\left(\frac{1+\alpha}{1-\alpha}\right)^{\frac{\lambda_{1}}{\lambda}} v^{\frac{-\lambda_{1}}{\lambda}}+\left(\frac{1+\alpha}{1-\alpha}\right)^{\frac{\lambda_{2}}{\lambda}} v^{\frac{-\lambda_{2}}{\lambda}}\right] d v \\
& =\frac{1}{\lambda(1+a)}\left[\left(\frac{1+a}{1-a}\right)^{\frac{\lambda_{1}}{\lambda}}+\left(\frac{1+a}{1-a}\right)^{\frac{\lambda_{2}}{\lambda}}\right] \frac{\pi}{\sin \left(\frac{\pi \lambda_{1}}{\lambda}\right)} \\
& -\frac{1}{\lambda(1+a)} \sum_{k=0}^{\infty} \int_{0}^{\frac{1+a}{1-a}}\left(v^{2 k}-v^{2 k+1}\right) \\
& \times\left[\left(\frac{1+a}{1-a}\right)^{\frac{\lambda_{1}}{\lambda}} v^{\frac{-\lambda_{1}}{\lambda}}+\left(\frac{1+a}{1-a}\right)^{\frac{\lambda_{2}}{\lambda}} v^{\frac{-\lambda_{2}}{\lambda}}\right] d v \\
& =\frac{1}{\lambda(1+\alpha)}\left[\left(\frac{1+\alpha}{1-\alpha}\right)^{\frac{\lambda_{1}}{\lambda}}+\left(\frac{1+\alpha}{1-\alpha}\right)^{\frac{\lambda_{2}}{\lambda}}\right] \frac{\pi}{\sin \left(\frac{\pi \lambda_{1}}{\lambda}\right)} \\
& -\frac{1}{\lambda(1+\alpha)} \sum_{k=0}^{\infty} \int_{0}^{\frac{1+\alpha}{1-\alpha}}(-1)^{k} v^{k} \\
& \times\left[\left(\frac{1+\alpha}{1-\alpha}\right)^{\frac{\lambda_{1}}{\lambda}} v^{\frac{\lambda_{2}}{\lambda}-1}+\left(\frac{1+\alpha}{1-\alpha}\right)^{\frac{\lambda_{2}}{\lambda}} v^{\frac{\lambda_{1}}{\lambda}-1}\right] d v \\
& =\frac{1}{1+\alpha}\left[\left(\frac{1+\alpha}{1-\alpha}\right)^{\frac{\lambda_{1}}{\lambda}}+\left(\frac{1+\alpha}{1-\alpha}\right)^{\frac{\lambda_{2}}{\lambda}}\right] \frac{\pi}{\lambda \sin \left(\frac{\pi \lambda_{1}}{\lambda}\right)} \\
& -\frac{1}{1+\alpha} \sum_{k=0}^{\infty}(-1)^{k}\left(\frac{1+\alpha}{1-\alpha}\right)^{k+1}\left(\frac{1}{\lambda k+\lambda_{2}}+\frac{1}{\lambda k+\lambda_{1}}\right) \text {. }
\end{aligned}
$$

(v) For $\lambda_{1}=\lambda_{2}=\frac{\lambda}{2} \in(0,1],-1<\alpha<1$, in view of (9), we find

$$
\begin{aligned}
k_{\alpha}\left(\frac{\lambda}{2}\right) & =\frac{2}{1+\alpha} \int_{0}^{1} \frac{t^{\frac{\lambda}{2}-1}}{\left(1+\frac{1-\alpha}{1+\alpha}\right) t^{\lambda}} d t \\
u=\left(\frac{1-\alpha}{1+\alpha}{ }^{\lambda}\right)^{\frac{1}{2}} & \frac{4\left(\frac{1+\alpha}{1-\alpha}\right)^{\frac{1}{2}}}{\lambda(1+\alpha)} \int_{0}^{\left(\frac{1-\alpha}{1+\alpha}\right)^{\frac{1}{2}}} \frac{1}{1+u^{2}} d u \\
& =\frac{4}{\lambda\left(1-\alpha^{2}\right)^{\frac{1}{2}}} \arctan \left(\frac{1-\alpha}{1+\alpha}\right)^{\frac{1}{2}} .
\end{aligned}
$$

We still have $k_{1}\left(\frac{\lambda}{2}\right)=\lim _{\alpha \rightarrow 1^{-}} k_{\alpha}\left(\frac{\lambda}{2}\right)=\frac{2}{\lambda}$.

(2) For fixed $x>0$, in view of $-1<\alpha \leq 1, \lambda>0$, we find that

$$
\begin{aligned}
k_{\lambda}(x, y) & =\frac{1}{x^{\lambda}+y^{\lambda}+\alpha\left|x^{\lambda}-y^{\lambda}\right|} \\
& = \begin{cases}\frac{1}{(1+\alpha) x^{\lambda}+(1-\alpha) y^{\lambda}}, & 0<y<x, \\
\frac{1}{(1-\alpha) x^{\lambda}+(1+\alpha) y^{\lambda}}, & y \geq x,\end{cases}
\end{aligned}
$$


is decreasing for $y>0$ and strictly decreasing for $y \in[x, \infty)$. In the same way, for fixed $y>0, k_{\lambda}(x, y)$ is decreasing for $x>0$ and strictly decreasing for $x \in[y, \infty)$.

Lemma 1 (cf. [29]) Suppose that $g(t)(>0)$ is decreasing in $\mathbf{R}_{+}$and strictly decreasing in $\left[n_{0}, \infty\right)\left(n_{0} \in \mathbf{N}\right)$, satisfying $\int_{0}^{\infty} g(t) d t \in \mathbf{R}_{+}$. We have

$$
\int_{1}^{\infty} g(t) d t<\sum_{n=1}^{\infty} g(n)<\int_{0}^{\infty} g(t) d t
$$

Lemma 2 Suppose that $U_{m}$ and $V_{n}$ are defined by (4) with $\mu_{1}, v_{1} \geq 1,-1<\alpha \leq 1,0<$ $\lambda_{1}, \lambda_{2} \leq 1, \lambda_{1}+\lambda_{2}=\lambda, K_{\lambda}(m, n)$, and $k_{\alpha}\left(\lambda_{1}\right)$ are indicated by (7) and (9). Define the following weight coefficients:

$$
\begin{aligned}
& \omega\left(\lambda_{2}, m\right):=\sum_{n=2}^{\infty} K_{\lambda}(m, n) \frac{v_{n} \ln ^{\lambda_{1}} U_{m}}{V_{n} \ln ^{1-\lambda_{2}} V_{n}}, \quad m \in \mathbf{N} \backslash\{1\}, \\
& \varpi\left(\lambda_{1}, n\right):=\sum_{m=2}^{\infty} K_{\lambda}(m, n) \frac{\mu_{m} \ln ^{\lambda_{2}} V_{n}}{U_{m} \ln ^{1-\lambda_{1}} U_{m}}, \quad n \in \mathbf{N} \backslash\{1\} .
\end{aligned}
$$

Then we have the following inequalities:

$$
\begin{array}{ll}
\omega\left(\lambda_{2}, m\right)<k_{\alpha}\left(\lambda_{1}\right) & \left(0<\lambda_{2} \leq 1, \lambda_{1}>0 ; m \in \mathbf{N} \backslash\{1\}\right), \\
\varpi\left(\lambda_{1}, n\right)<k_{\alpha}\left(\lambda_{1}\right) & \left(0<\lambda_{1} \leq 1, \lambda_{2}>0 ; n \in \mathbf{N} \backslash\{1\}\right) .
\end{array}
$$

Proof We set $\mu(t):=\mu_{m}, t \in(m-1, m](m \in \mathbf{N}) ; v(t):=v_{n}, t \in(n-1, n](n \in \mathbf{N})$, and

$$
U(x):=\int_{0}^{x} \mu(t) d t \quad(x \geq 0), \quad V(y):=\int_{0}^{y} v(t) d t \quad(y \geq 0) .
$$

Then it follows that $U(m)=U_{m}, V(n)=V_{n}(m, n \in \mathbf{N}) . U^{\prime}(x)=\mu(x)=\mu_{m}$, for $x \in(m-1, m)$ $(m \in \mathbf{N}) ; V^{\prime}(y)=v(y)=v_{n}$, for $y \in(n-1, n)(n \in \mathbf{N})$. Since $V(y)$ is strictly increasing in $(n-1, n], 0<\lambda_{2} \leq 1, \lambda_{1}>0$, in view of Example 1(2) and Lemma 1, we find

$$
\begin{aligned}
\omega\left(\lambda_{2}, m\right) & =\sum_{n=2}^{\infty} \int_{n-1}^{n} k_{\lambda}\left(\ln U_{m}, \ln V_{n}\right) \frac{\ln ^{\lambda_{1}} U_{m}}{V_{n} \ln ^{1-\lambda_{2}} V_{n}} V^{\prime}(y) d y \\
& <\sum_{n=2}^{\infty} \int_{n-1}^{n} k_{\lambda}\left(\ln U_{m}, \ln V(y)\right) \frac{\ln ^{\lambda_{1}} U_{m}}{V(y) \ln ^{1-\lambda_{2}} V(y)} V^{\prime}(y) d y \\
& =\int_{1}^{\infty} k_{\lambda}\left(\ln U_{m}, \ln V(y)\right) \frac{\ln ^{\lambda_{1}} U_{m}}{V(y) \ln ^{1-\lambda_{2}} V(y)} V^{\prime}(y) d y
\end{aligned}
$$

Setting $t=\frac{\ln V(y)}{\ln U_{m}}$, we obtain $\frac{1}{V(y)} V^{\prime}(y) d y=\ln U_{m} d t$ and

$$
\omega\left(\lambda_{2}, m\right)<\int_{\frac{\ln V(1)}{\ln U_{m}}}^{\frac{\ln V(\infty)}{\ln u_{m}}} k_{\lambda}(1, t) t^{\lambda_{2}-1} d t \leq \int_{0}^{\infty} k_{\lambda}(1, t) t^{\lambda_{2}-1} d t=k_{\alpha}\left(\lambda_{1}\right) .
$$

Hence, we have (17). In the same way, we have (18). 
Note We do not need the condition $\lambda_{1} \leq 1\left(\lambda_{2} \leq 1\right)$ to obtain (17) ((18)).

Lemma 3 As regards the assumptions of Lemma 2, if $U(\infty)=V(\infty)=\infty$, there exist $m_{0}, n_{0} \in \mathbf{N} \backslash\{1\}$, such that $\mu_{m} \geq \mu_{m+1}\left(m \in\left\{m_{0}, m_{0}+1, \ldots\right\}\right), v_{n} \geq v_{n+1}\left(n \in\left\{n_{0}, n_{0}+1, \ldots\right\}\right)$, then:

(i) For $m, n \in \mathbf{N} \backslash\{1\}$, we have

$$
\begin{array}{lc}
k_{\alpha}\left(\lambda_{1}\right)\left(1-\theta\left(\lambda_{2}, m\right)\right)<\omega\left(\lambda_{2}, m\right) & \left(0<\lambda_{2} \leq 1, \lambda_{1}>0\right), \\
k_{\alpha}\left(\lambda_{1}\right)\left(1-\vartheta\left(\lambda_{1}, n\right)\right)<\varpi\left(\lambda_{1}, n\right) & \left(0<\lambda_{1} \leq 1, \lambda_{2}>0\right),
\end{array}
$$

where

$$
\begin{aligned}
\theta\left(\lambda_{2}, m\right) & :=\frac{1}{k_{\alpha}\left(\lambda_{1}\right)} \int_{0}^{\frac{\ln V_{n_{0}}}{\ln u_{m}}} \frac{t^{\lambda_{2}-1} d t}{1+t^{\lambda}+\alpha\left|1-t^{\lambda}\right|} \\
& =O\left(\frac{1}{\ln ^{\lambda_{2}} U_{m}}\right) \in(0,1), \\
\vartheta\left(\lambda_{1}, n\right) & :=\frac{1}{k_{\alpha}\left(\lambda_{1}\right)} \int_{0}^{\frac{\ln U_{m_{0}}}{\ln V_{n}}} \frac{t^{\lambda_{2}-1} d t}{1+t^{\lambda}+\alpha\left|1-t^{\lambda}\right|} \\
& =O\left(\frac{1}{\ln ^{\lambda_{1}} V_{n}}\right) \in(0,1) .
\end{aligned}
$$

(ii) For any $b>0$, we have

$$
\begin{aligned}
& \sum_{m=2}^{\infty} \frac{\mu_{m}}{U_{m} \ln ^{1+b} U_{m}}=\frac{1}{b}\left(\frac{1}{\ln ^{b} U_{m_{0}}}+b O_{1}(1)\right), \\
& \sum_{n=2}^{\infty} \frac{v_{n}}{V_{n} \ln ^{1+b} V_{n}}=\frac{1}{b}\left(\frac{1}{\ln ^{b} V_{n_{0}}}+b O_{2}(1)\right) .
\end{aligned}
$$

Proof Since $v_{n} \geq v_{n+1}\left(n \geq n_{0}\right), 0<\lambda_{2} \leq 1, \lambda_{1}>0$, and $V(\infty)=\infty$, by Example 1(2), Lemma 1 , and (23), we find

$$
\begin{aligned}
\omega\left(\lambda_{2}, m\right) & \geq \sum_{n=n_{0}}^{\infty} K_{\lambda}(m, n) \frac{v_{n+1} \ln ^{\lambda_{1}} U_{m}}{V_{n} \ln ^{1-\lambda_{2}} V_{n}} \\
& =\sum_{n=n_{0}}^{\infty} \int_{n}^{n+1} k_{\lambda}\left(\ln U_{m}, \ln V_{n}\right) \frac{\ln ^{\lambda_{1}} U_{m}}{V_{n} \ln ^{1-\lambda_{2}} V_{n}} V^{\prime}(y) d y \\
& >\sum_{n=n_{0}}^{\infty} \int_{n}^{n+1} k_{\lambda}\left(\ln U_{m}, \ln V(y)\right) \frac{\ln ^{\lambda_{1}} U_{m}}{V(y) \ln ^{1-\lambda_{2}} V(y)} V^{\prime}(y) d y \\
& =\int_{n_{0}}^{\infty} k_{\lambda}\left(\ln U_{m}, \ln V(y)\right) \frac{\ln ^{\lambda_{1}} U_{m}}{V(y) \ln ^{1-\lambda_{2}} V(y)} V^{\prime}(y) d y \\
& t=\frac{\ln V(y)}{\ln u_{m}} \int_{\frac{\ln V_{n_{0}}}{\ln u_{m}}}^{\infty} \frac{t^{\lambda_{2}-1} d t}{1+t^{\lambda}+\alpha\left|1-t^{\lambda}\right|}=k_{\alpha}\left(\lambda_{1}\right)\left(1-\theta\left(\lambda_{2}, m\right)\right) .
\end{aligned}
$$


We obtain

$$
0<\theta\left(\lambda_{2}, m\right) \leq \frac{1}{k_{\alpha}\left(\lambda_{1}\right)} \int_{0}^{\frac{\ln V_{n_{0}}}{\ln u_{m}}} t^{\lambda_{2}-1} d t=\frac{1}{\lambda_{2} k_{\alpha}\left(\lambda_{1}\right)}\left(\frac{\ln V_{n_{0}}}{\ln U_{m}}\right)^{\lambda_{2}},
$$

namely, $\theta\left(\lambda_{2}, m\right)=O\left(\frac{1}{\ln ^{2} 2 U_{m}}\right)$. Hence we have (21). In the same way, we obtain (22).

For $b>0$, we find

$$
\begin{aligned}
\sum_{m=2}^{\infty} \frac{\mu_{m}}{U_{m} \ln ^{1+b} U_{m}} & =\sum_{m=2}^{m_{0}} \frac{\mu_{m}}{U_{m} \ln ^{1+b} U_{m}}+\sum_{m=m_{0}+1}^{\infty} \frac{\mu_{m}}{U_{m} \ln ^{1+b} U_{m}} \\
& =\sum_{m=2}^{m_{0}} \frac{\mu_{m}}{U_{m} \ln ^{1+b} U_{m}}+\sum_{m=m_{0}+1}^{\infty} \int_{m-1}^{m} \frac{U^{\prime}(x)}{U_{m} \ln ^{1+b} U_{m}} d x \\
& <\sum_{m=2}^{m_{0}} \frac{\mu_{m}}{U_{m} \ln ^{1+b} U_{m}}+\sum_{m=m_{0}+1}^{\infty} \int_{m-1}^{m} \frac{U^{\prime}(x)}{U(x) \ln ^{1+b} U(x)} d x \\
& =\sum_{m=2}^{m_{0}} \frac{\mu_{m}}{U_{m} \ln ^{1+b} U_{m}}+\int_{m_{0}}^{\infty} \frac{d U(x)}{U(x) \ln ^{1+b} U(x)} \\
& =\frac{1}{b}\left(\frac{1}{\ln ^{b} U_{m_{0}}}+b \sum_{m=2}^{m} \frac{\mu_{m}}{U_{m} \ln ^{1+b} U_{m}}\right), \\
\sum_{m=2}^{\infty} \frac{\mu_{m}}{U_{m} \ln ^{1+b} U_{m}} & \geq \sum_{m=m_{0}}^{\infty} \frac{\mu_{m}}{U_{m} \ln ^{1+b} U_{m}} \geq \sum_{m=m_{0}}^{\infty} \frac{\mu_{m+1}}{U_{m} \ln ^{1+b} U_{m}} \\
& =\sum_{m=m_{0}}^{\infty} \int_{m}^{m+1} \frac{U^{\prime}(x) d x}{U_{m} \ln ^{1+b} U_{m}}>\sum_{m=m_{0}}^{\infty} \int_{m}^{m+1} \frac{U^{\prime}(x) d x}{U(x) \ln ^{1+b} U(x)} \\
& =\int_{m_{0}}^{\infty} \frac{d U(x)}{U_{(x) \ln ^{1+b} U(x)} U^{1+b}} \frac{1}{b \ln ^{b} U_{m_{0}}} .
\end{aligned}
$$

Hence we have (25). In the same way, we have (26).

Lemma 4 If $-1<\alpha \leq 1,0<\lambda_{1}, \lambda_{2} \leq 1, \lambda_{1}+\lambda_{2}=\lambda, k_{\alpha}\left(\lambda_{1}\right)$ is indicated in (9), then for $0<$ $\delta<\min \left\{\lambda_{1}, \lambda_{2}\right\}$, we have

$$
k_{\alpha}\left(\lambda_{1} \pm \delta\right)=k_{\alpha}\left(\lambda_{1}\right)+o(1) \quad\left(\delta \rightarrow 0^{+}\right)
$$

Proof We find for $0<\delta<\min \left\{\lambda_{1}, \lambda_{2}\right\}$,

$$
\begin{aligned}
& \left|k_{\alpha}\left(\lambda_{1}+\delta\right)-k_{\alpha}\left(\lambda_{1}\right)\right| \\
& \quad \leq \int_{0}^{\infty} \frac{t^{\lambda_{1}-1}\left|t^{\delta}-1\right|}{t^{\lambda}+1+\alpha\left|t^{\lambda}-1\right|} d t \\
& \quad=\int_{0}^{1} \frac{t^{\lambda_{1}-1}\left(1-t^{\delta}\right) d t}{1+\alpha+(1-\alpha) t^{\lambda}}+\int_{1}^{\infty} \frac{t^{\lambda_{1}-1}\left(t^{\delta}-1\right) d t}{1-\alpha+(1+\alpha) t^{\lambda}} \\
& \quad \leq \frac{1}{1+\alpha}\left[\int_{0}^{1} t^{\lambda_{1}-1}\left(1-t^{\delta}\right) d t+\int_{1}^{\infty} \frac{t^{\lambda_{1}-1}\left(t^{\delta}-1\right)}{t^{\lambda}} d t\right] \\
& \quad=\frac{1}{1+\alpha}\left(\frac{1}{\lambda_{1}}-\frac{1}{\lambda_{1}+\delta}+\frac{1}{\lambda_{2}-\delta}-\frac{1}{\lambda_{2}}\right) \rightarrow 0 \quad\left(\delta \rightarrow 0^{+}\right) .
\end{aligned}
$$


In the same way, we find

$$
\begin{aligned}
& \left|k_{\alpha}\left(\lambda_{1}-\delta\right)-k_{\alpha}\left(\lambda_{1}\right)\right| \\
& \quad \leq \frac{1}{1+\alpha}\left[\int_{0}^{1} t^{\lambda_{1}-1}\left(t^{-\delta}-1\right) d t+\int_{1}^{\infty} \frac{t^{\lambda_{1}-1}\left(1-t^{-\delta}\right)}{t^{\lambda}} d t\right] \\
& \quad \leq \frac{1}{1+\alpha}\left(\frac{1}{\lambda_{1}-\delta}-\frac{1}{\lambda_{1}}+\frac{1}{\lambda_{2}}-\frac{1}{\lambda_{2}+\delta}\right) \rightarrow 0 \quad\left(\delta \rightarrow 0^{+}\right),
\end{aligned}
$$

and then we have (27).

\section{Main results}

In the following, we agree that $\mu_{1}, v_{1} \geq 1, \mu_{i}, v_{j}>0(i, j \in \mathbf{N} \backslash\{1\}), U_{m}$ and $V_{n}$ are defined by (4), $-1<\alpha \leq 1,0<\lambda_{1}, \lambda_{2} \leq 1, \lambda_{1}+\lambda_{2}=\lambda, K_{\lambda}(m, n)$, and $k_{\alpha}\left(\lambda_{1}\right)$ are indicated by (7) and (9), $p>1, \frac{1}{p}+\frac{1}{q}=1, a_{m}, b_{n} \geq 0(m, n \in \mathbf{N} \backslash\{1\})$,

$$
\|a\|_{p, \Phi_{\lambda}}:=\left(\sum_{m=2}^{\infty} \Phi_{\lambda}(m) a_{m}^{p}\right)^{\frac{1}{p}}, \quad\|b\|_{q, \Psi_{\lambda}}:=\left(\sum_{n=2}^{\infty} \Psi_{\lambda}(n) b_{n}^{q}\right)^{\frac{1}{q}},
$$

where

$$
\begin{aligned}
& \Phi_{\lambda}(m):=\left(\frac{U_{m}}{\mu_{m}}\right)^{p-1}\left(\ln U_{m}\right)^{p\left(1-\lambda_{1}\right)-1}, \\
& \Psi_{\lambda}(n):=\left(\frac{V_{n}}{v_{n}}\right)^{q-1}\left(\ln V_{n}\right)^{q\left(1-\lambda_{2}\right)-1} \quad(m, n \in \mathbf{N} \backslash\{1\}) .
\end{aligned}
$$

Theorem 1 If $0<\|a\|_{p, \Phi_{\lambda}},\|b\|_{q, \Psi_{\lambda}}<\infty$, then we have the following equivalent inequalities:

$$
\begin{aligned}
& I:=\sum_{n=2}^{\infty} \sum_{m=2}^{\infty} K_{\lambda}(m, n) a_{m} b_{n}<k_{\alpha}\left(\lambda_{1}\right)\|a\|_{p, \Phi_{\lambda}}\|b\|_{q, \Psi_{\lambda}}, \\
& J:=\left[\sum_{n=2}^{\infty} \frac{v_{n}}{V_{n}}\left(\ln V_{n}\right)^{p \lambda_{2}-1}\left(\sum_{m=2}^{\infty} K_{\lambda}(m, n) a_{m}\right)^{p}\right]^{\frac{1}{p}}<k_{\alpha}\left(\lambda_{1}\right)\|a\|_{p, \Phi_{\lambda}} .
\end{aligned}
$$

In particular, for $\lambda_{1}=\lambda_{2}=\frac{\lambda}{2} \in(0,1]$, the constant factor $k_{\alpha}\left(\lambda_{1}\right)$ in the above inequalities is expressed in the following form:

$$
k_{\alpha}\left(\frac{\lambda}{2}\right)=\frac{4}{\lambda\left(1-\alpha^{2}\right)^{\frac{1}{2}}} \arctan \left(\frac{1-\alpha}{1+\alpha}\right)^{\frac{1}{2}} .
$$

Proof By Hölder's inequality with weight (cf. [32]) and (16), we have

$$
\begin{aligned}
\left(\sum_{m=2}^{\infty} K_{\lambda}(m, n) a_{m}\right)^{p} & =\left[\sum_{m=2}^{\infty} K_{\lambda}(m, n)\left(\frac{U_{m}^{1 / q}\left(\ln U_{m}\right)^{\left(1-\lambda_{1}\right) / q}}{\left(\ln V_{n}\right)^{\left(1-\lambda_{2}\right) / p} \mu_{m}^{1 / q}} a_{m}\right)\left(\frac{\left(\ln V_{n}\right)^{\left(1-\lambda_{2}\right) / p} \mu_{m}^{1 / q}}{U_{m}^{1 / q}\left(\ln U_{m}\right)^{\left(1-\lambda_{1}\right) / q}}\right)\right]^{p} \\
& \leq \sum_{m=2}^{\infty} K_{\lambda}(m, n) \frac{U_{m}^{p-1}\left(\ln U_{m}\right)^{\left(1-\lambda_{1}\right) p / q}}{\left(\ln V_{n}\right)^{1-\lambda_{2}} \mu_{m}^{p / q}} a_{m}^{p}
\end{aligned}
$$




$$
\begin{aligned}
\times\left[\sum_{m=2}^{\infty} K_{\lambda}(m, n) \frac{\left(\ln V_{n}\right)^{\left(1-\lambda_{2}\right)(q-1)} \mu_{m}}{U_{m}\left(\ln U_{m}\right)^{1-\lambda_{1}}}\right]^{p-1} \\
=\frac{\left(\varpi\left(\lambda_{1}, n\right)\right)^{p-1} V_{n}}{\left(\ln V_{n}\right)^{p \lambda_{2}-1} v_{n}} \sum_{m=2}^{\infty} K_{\lambda}(m, n) \frac{v_{n} U_{m}^{p-1}\left(\ln U_{m}\right)^{\left(1-\lambda_{1}\right)(p-1)} a_{m}^{p}}{V_{n}\left(\ln V_{n}\right)^{1-\lambda_{2}} \mu_{m}^{p-1}} .
\end{aligned}
$$

Then by (18), we obtain

$$
\begin{aligned}
J & \leq\left(k_{\alpha}\left(\lambda_{1}\right)\right)^{\frac{1}{q}}\left[\sum_{n=2}^{\infty} \sum_{m=2}^{\infty} K_{\lambda}(m, n) \frac{v_{n} U_{m}^{p-1}\left(\ln U_{m}\right)^{\left(1-\lambda_{1}\right)(p-1)} a_{m}^{p}}{V_{n}\left(\ln V_{n}\right)^{1-\lambda_{2}} \mu_{m}^{p-1}}\right]^{\frac{1}{p}} \\
& =\left(k_{\alpha}\left(\lambda_{1}\right)\right)^{\frac{1}{q}}\left[\sum_{m=2}^{\infty} \sum_{n=2}^{\infty} K_{\lambda}(m, n) \frac{v_{n} U_{m}^{p-1}\left(\ln U_{m}\right)^{\left(1-\lambda_{1}\right)(p-1)} a_{m}^{p}}{V_{n}\left(\ln V_{n}\right)^{1-\lambda_{2}} \mu_{m}^{p-1}}\right]^{\frac{1}{p}} \\
& =\left(k_{\alpha}\left(\lambda_{1}\right)\right)^{\frac{1}{q}}\left[\sum_{m=2}^{\infty} \omega\left(\lambda_{2}, m\right) \frac{\left(\ln U_{m}\right)^{p\left(1-\lambda_{1}\right)-1}}{U_{m}^{1-p} \mu_{m}^{p-1}} a_{m}^{p}\right]^{\frac{1}{p}} .
\end{aligned}
$$

Hence, by (17), we have (29).

By Hölder's inequality (cf. [32]), we have

$$
\begin{aligned}
I= & \sum_{n=2}^{\infty}\left[\left(\frac{v_{n}}{V_{n}}\right)^{1 / p}\left(\ln V_{n}\right)^{\lambda_{2}-\frac{1}{p}} \sum_{m=2}^{\infty} K_{\lambda}(m, n) a_{m}\right] \\
& \times\left[\left(\frac{v_{n}}{V_{n}}\right)^{-1 / p}\left(\ln V_{n}\right)^{\frac{1}{p}-\lambda_{2}} b_{n}\right] \leq J\|b\|_{q, \Psi_{\lambda}}
\end{aligned}
$$

and then by (29), we have (28).

On the other hand, assuming that (29) is valid, setting

$$
b_{n}:=\frac{v_{n}}{V_{n}}\left(\ln V_{n}\right)^{p \lambda_{2}-1}\left(\sum_{m=2}^{\infty} K_{\lambda}(m, n) a_{m}\right)^{p-1}, \quad n \in \mathbf{N} \backslash\{1\},
$$

we find $J^{p}=\|b\|_{q, \Psi_{\lambda}}^{q}$. If $J=0$, then (29) is trivially valid; if $J=\infty$, then by (31) and (17), it is impossible; if $0<J<\infty$, then by (28), it follows that

$$
\begin{aligned}
& \|b\|_{q, \Psi_{\lambda}}^{q}=J^{p}=I<k_{\alpha}\left(\lambda_{1}\right)\|a\|_{p, \Phi_{\lambda}}\|b\|_{q, \Psi_{\lambda},}, \\
& \|b\|_{q, \Psi_{\lambda}}^{q-1}=J<k_{\alpha}\left(\lambda_{1}\right)\|a\|_{p, \Phi_{\lambda}},
\end{aligned}
$$

namely, (29) follows, which is equivalent to (28).

Theorem 2 With regards the assumptions of Theorem 1 , if $U(\infty)=V(\infty)=\infty$, there exist $m_{0}, n_{0} \in \mathbf{N} \backslash\{1\}$, such that $\mu_{m} \geq \mu_{m+1}\left(m \in\left\{m_{0}, m_{0}+1, \ldots\right\}\right), v_{n} \geq v_{n+1}\left(n \in\left\{n_{0}, n_{0}+1, \ldots\right\}\right)$, then the constant factor $k_{\alpha}\left(\lambda_{1}\right)$ in (28) and (29) is the best possible.

Proof For $\varepsilon \in\left(0, p \lambda_{1}\right)$, we set $\tilde{\lambda}_{1}=\lambda_{1}-\frac{\varepsilon}{p}(\in(0,1)), \tilde{\lambda}_{2}=\lambda_{2}+\frac{\varepsilon}{p}(>0), \tilde{a}=\left\{\tilde{a}_{m}\right\}_{m=2}^{\infty}, \tilde{b}=\left\{\tilde{b}_{n}\right\}_{n=2}^{\infty}$, where

$$
\begin{aligned}
& \tilde{a}_{m}:=\frac{\mu_{m}}{U_{m}} \ln ^{\tilde{\lambda}_{1}-1} U_{m}=\frac{\mu_{m}}{U_{m}} \ln ^{\lambda_{1}-\frac{\varepsilon}{p}-1} U_{m}, \\
& \tilde{b}_{n}:=\frac{v_{n}}{V_{n}} \ln ^{\tilde{\lambda}_{2}-\varepsilon-1} V_{n}=\frac{v_{n}}{V_{n}} \ln ^{\lambda_{2}-\frac{\varepsilon}{q}-1} V_{n} .
\end{aligned}
$$


Then by (25), (26), and (22), we find

$$
\begin{aligned}
\|\tilde{a}\|_{p, \Phi_{\lambda}}\|\tilde{b}\|_{q, \Psi_{\lambda}}=\left(\sum_{m=2}^{\infty} \frac{\mu_{m}}{U_{m} \ln ^{1+\varepsilon} U_{m}}\right)^{\frac{1}{p}}\left(\sum_{n=2}^{\infty} \frac{v_{n}}{V_{n} \ln ^{1+\varepsilon} V_{n}}\right)^{\frac{1}{q}} \\
\quad=\frac{1}{\varepsilon}\left(\frac{1}{\ln ^{\varepsilon} U_{m_{0}}}+\varepsilon O_{1}(1)\right)^{\frac{1}{p}}\left(\frac{1}{\ln ^{\varepsilon} V_{n_{0}}}+\varepsilon O_{2}(1)\right)^{\frac{1}{q}} \\
\tilde{I}:=\sum_{n=2}^{\infty} \sum_{m=2}^{\infty} K_{\lambda}(m, n) \tilde{a}_{m} \tilde{b}_{n} \\
=\sum_{n=2}^{\infty}\left(\sum_{m=2}^{\infty} K_{\lambda}(m, n) \frac{\mu_{m} \ln ^{\tilde{\lambda}_{2}} V_{n}}{U_{m} \ln ^{1-\tilde{\lambda}_{1}} U_{m}}\right) \frac{v_{n}}{V_{n} \ln ^{\varepsilon+1} V_{n}} \\
=\sum_{n=2}^{\infty} \frac{\varpi\left(\tilde{\lambda}_{1}, n\right) v_{n}}{V_{n} \ln ^{\varepsilon+1} V_{n}} \geq k_{\alpha}\left(\tilde{\lambda}_{1}\right) \sum_{n=2}^{\infty}\left(1-O\left(\frac{1}{\ln ^{\tilde{\lambda}_{1}} V_{n}}\right)\right) \frac{v_{n}}{V_{n} \ln ^{\varepsilon+1} V_{n}} \\
=k_{\alpha}\left(\tilde{\lambda}_{1}\right)\left[\sum_{n=2}^{\infty} \frac{v_{n}}{V_{n} \ln ^{\varepsilon+1} V_{n}}-\sum_{n=2}^{\infty} O\left(\frac{v_{n}}{V_{n}\left(\ln V_{n}\right)^{\left(\frac{\varepsilon}{q}+\lambda_{1}\right)+1}}\right)\right] \\
=\frac{1}{\varepsilon} k_{\alpha}\left(\tilde{\lambda}_{1}\right)\left[\frac{1}{\ln ^{\varepsilon} V_{n_{0}}}+\varepsilon\left(O_{2}(1)-O(1)\right)\right] .
\end{aligned}
$$

If there exists a positive constant $K \leq k_{\alpha}\left(\lambda_{1}\right)$, such that (28) is valid when replacing $k_{\alpha}\left(\lambda_{1}\right)$ by $K$, then, in particular, we have $\varepsilon \tilde{I}<\varepsilon K\|\tilde{a}\|_{p, \Phi_{\lambda}}\|\tilde{b}\|_{q, \Psi_{\lambda}}$, namely

$$
\begin{aligned}
& k_{\alpha}\left(\lambda_{1}-\frac{\varepsilon}{p}\right)\left[\frac{1}{\ln ^{\varepsilon} V_{n_{0}}}+\varepsilon\left(O_{2}(1)-O(1)\right)\right] \\
& \quad<K\left(\frac{1}{\ln ^{\varepsilon} U_{m_{0}}}+\varepsilon O_{1}(1)\right)^{\frac{1}{p}}\left(\frac{1}{\ln ^{\varepsilon} V_{n_{0}}}+\varepsilon O_{2}(1)\right)^{\frac{1}{q}} .
\end{aligned}
$$

By (27), it follows that $k_{\alpha}\left(\lambda_{1}\right) \leq K\left(\varepsilon \rightarrow 0^{+}\right)$. Hence, $K=k_{\alpha}\left(\lambda_{1}\right)$ is the best possible constant factor of (28).

The constant factor $k_{\alpha}\left(\lambda_{1}\right)$ in (29) is still the best possible. Otherwise, we would reach a contradiction by (32) that the constant factor in (28) is not the best possible.

For $p>1$, we find $\Psi_{\lambda}^{1-p}(n)=\frac{v_{n}}{V_{n}}\left(\ln V_{n}\right)^{p \lambda_{2}-1}(n \in \mathbf{N} \backslash\{1\})$ and define the following normed spaces:

$$
\begin{aligned}
& l_{p, \Phi_{\lambda}}:=\left\{a=\left\{a_{m}\right\}_{m=2}^{\infty} ;\|a\|_{p, \Phi_{\lambda}}<\infty\right\}, \\
& l_{q, \Psi_{\lambda}}:=\left\{b=\left\{b_{n}\right\}_{n=2}^{\infty} ;\|b\|_{q, \Psi_{\lambda}}<\infty\right\}, \\
& l_{p, \Psi_{\lambda}^{1-p}}:=\left\{c=\left\{c_{n}\right\}_{n=2}^{\infty} ;\|c\|_{\left.p, \Psi_{\lambda}^{1-p}<\infty\right\} .}\right.
\end{aligned}
$$

Assuming that $a=\left\{a_{m}\right\}_{m=2}^{\infty} \in l_{p, \Phi_{\lambda}}$, setting

$$
c=\left\{c_{n}\right\}_{n=2}^{\infty}, \quad c_{n}:=\sum_{m=2}^{\infty} K_{\lambda}(m, n) a_{m}, \quad n \in \mathbf{N}
$$

we can rewrite (29) as $\|c\|_{p, \Psi_{\lambda}^{1-p}}<k_{\alpha}\left(\lambda_{1}\right)\|a\|_{p, \Phi_{\lambda}}<\infty$, namely, $c \in l_{p, \Psi_{\lambda}^{1-p}}$. 
Definition 1 Define a Hardy-Mulholland-type operator $T: l_{p, \Phi_{\lambda}} \rightarrow l_{p, \Psi_{\lambda}^{1-p}}$ as follows: for any $a=\left\{a_{m}\right\}_{m=2}^{\infty} \in l_{p, \Phi_{\lambda}}$, there exists a unique representation $T a=c \in l_{p, \Psi_{\lambda}^{1-p}}$. Define the formal inner product of $T a$ and $b=\left\{b_{n}\right\}_{n=2}^{\infty} \in l_{q, \Psi_{\lambda}}$ as follows:

$$
(T a, b):=\sum_{n=2}^{\infty}\left(\sum_{m=2}^{\infty} K_{\lambda}(m, n) a_{m}\right) b_{n} .
$$

Then we can rewrite (28) and (29) as follows:

$$
\begin{aligned}
& (T a, b)<k_{\alpha}\left(\lambda_{1}\right)\|a\|_{p, \Phi_{\lambda}}\|b\|_{q, \Psi_{\lambda}}, \\
& \|T a\|_{p, \Psi_{\lambda}^{1-p}}<k_{\alpha}\left(\lambda_{1}\right)\|a\|_{p, \Phi_{\lambda}} .
\end{aligned}
$$

Define the norm of operator $T$ as follows:

$$
\|T\|:=\sup _{a(\neq \theta) \in l_{p, \Phi_{\lambda}}} \frac{\|T a\|_{p, \Psi_{\lambda}^{1-p}}}{\|a\|_{p, \Phi_{\lambda}}} .
$$

Then by (39), it follows that $\|T\| \leq k_{\alpha}\left(\lambda_{1}\right)$. In view of Theorem 2 , the constant factor in (39) is the best possible, we have

$$
\|T\|=k_{\alpha}\left(\lambda_{1}\right)=\int_{0}^{1} \frac{t^{\lambda_{1}-1}+t^{\lambda_{2}-1}}{1+\alpha+(1-\alpha) t^{\lambda}} d t .
$$

Remark 1 (i) For $\lambda=1, \lambda_{1}=\frac{1}{q}, \lambda_{2}=\frac{1}{p}$, (28) reduces to

$$
\sum_{n=2}^{\infty} \sum_{m=2}^{\infty} \frac{a_{m} b_{n}}{\ln U_{m} V_{n}+\alpha\left|\ln \frac{U_{m}}{V_{n}}\right|}<k_{\alpha}\left(\frac{1}{q}\right)\left(\sum_{m=2}^{\infty} \frac{a_{m}^{p}}{\mu_{m}^{p-1}}\right)^{\frac{1}{p}}\left(\sum_{n=2}^{\infty} \frac{b_{n}^{q}}{v_{n}^{q-1}}\right)^{\frac{1}{q}} .
$$

In particular, for $\alpha=0$, we have the following simple Hardy-Mulholland-type inequality:

$$
\sum_{n=2}^{\infty} \sum_{m=2}^{\infty} \frac{a_{m} b_{n}}{\ln U_{m} V_{n}}<\frac{\pi}{\sin (\pi / p)}\left(\sum_{m=2}^{\infty} \frac{a_{m}^{p}}{\mu_{m}^{p-1}}\right)^{\frac{1}{p}}\left(\sum_{n=2}^{\infty} \frac{b_{n}^{q}}{v_{n}^{q-1}}\right)^{\frac{1}{q}},
$$

which is an extension of (3); for $\alpha=1$, we have another simple Hardy-Mulholland-type inequality:

$$
\sum_{n=2}^{\infty} \sum_{m=2}^{\infty} \frac{a_{m} b_{n}}{\max \left\{\ln U_{m}, \ln V_{n}\right\}}<p q\left(\sum_{m=2}^{\infty} \frac{a_{m}^{p}}{\mu_{m}^{p-1}}\right)^{\frac{1}{p}}\left(\sum_{n=2}^{\infty} \frac{b_{n}^{q}}{v_{n}^{q-1}}\right)^{\frac{1}{q}} .
$$

Hence, inequality (28) is a relation between (42) and (43).

(ii) For $\alpha=1$ in (28) and (29), in view of (9), we have the following equivalent HardyMulholland-type inequalities with parameters:

$$
\sum_{n=2}^{\infty} \sum_{m=2}^{\infty} \frac{a_{m} b_{n}}{\max \left\{\ln ^{\lambda} U_{m}, \ln ^{\lambda} V_{n}\right\}}<\frac{\lambda}{\lambda_{1} \lambda_{2}}\|a\|_{p, \Phi_{\lambda}}\|b\|_{q, \Psi_{\lambda}}
$$




$$
\begin{aligned}
& {\left[\sum_{n=2}^{\infty} \frac{v_{n}}{V_{n}}\left(\ln V_{n}\right)^{p \lambda_{2}-1}\left(\sum_{m=2}^{\infty} \frac{a_{m}}{\max \left\{\ln ^{\lambda} U_{m}, \ln ^{\lambda} V_{n}\right\}}\right)^{p}\right]^{\frac{1}{p}}} \\
& \quad<\frac{\lambda}{\lambda_{1} \lambda_{2}}\|a\|_{p, \Phi_{\lambda} .}
\end{aligned}
$$

(iii) For $\alpha=0$ in (28) and (29), in view of (11), we have another equivalent HardyMulholland-type inequalities with parameters:

$$
\begin{aligned}
& \sum_{n=2}^{\infty} \sum_{m=2}^{\infty} \frac{a_{m} b_{n}}{\ln ^{\lambda} U_{m}+\ln ^{\lambda} V_{n}}<\frac{\pi}{\lambda \sin \left(\frac{\pi \lambda_{1}}{\lambda}\right)}\|a\|_{p, \Phi_{\lambda}}\|b\|_{q, \Psi_{\lambda}}, \\
& {\left[\sum_{n=2}^{\infty} \frac{v_{n}}{V_{n}}\left(\ln V_{n}\right)^{p \lambda_{2}-1}\left(\sum_{m=2}^{\infty} \frac{a_{m}}{\ln ^{\lambda} U_{m}+\ln ^{\lambda} V_{n}}\right)^{p}\right]^{\frac{1}{p}}} \\
& <\frac{\pi}{\lambda \sin \left(\frac{\pi \lambda_{1}}{\lambda}\right)}\|a\|_{p, \Phi_{\lambda}} .
\end{aligned}
$$

In view of Theorem 2 , the constant factors in the above inequalities are all the best possible. Inequality (28) is also a relation between the two Hardy-Mulholland-type inequalities (46) and (44) with parameters.

\section{Competing interests}

The authors declare that they have no competing interests.

\section{Authors' contributions}

BY carried out the mathematical studies, participated in the sequence alignment and drafted the manuscript. QC and YS participated in the design of the study and performed the numerical analysis. All authors read and approved the final manuscript.

\section{Author details}

'Department of Computer Science, Guangdong University of Education, Guangzhou, Guangdong 51003, P.R. China. ${ }^{2}$ Normal College of Jishou University, Jishou, Hunan 416000, P.R. China. ${ }^{3}$ Department of Mathematics, Guangdong University of Education, Guangzhou, Guangdong 51003, P.R. China.

\section{Acknowledgements}

This work is supported by the National Natural Science Foundation of China (No. 61370186), Appropriative Researching Fund for Professors and Doctors, Guangdong University of Education (No. 2015ARF25) and Hunan Province Natural Science Foundation (No. 2015JJ4041).

Received: 13 December 2015 Accepted: 12 February 2016 Published online: 24 February 2016

\section{References}

1. Hardy, GH, Littlewood, JE, Pólya, G: Inequalities. Cambridge University Press, Cambridge (1934)

2. Mitrinović, DS, Pečarić, JE, Fink, AM: Inequalities Involving Functions and Their Integrals and Derivatives. Kluwer Academic, Boston (1991)

3. Yang, BC: Hilbert-Type Integral Inequalities. Bentham Science Publishers, Sharjah (2009)

4. Yang, BC: Discrete Hilbert-Type Inequalities. Bentham Science Publishers, Sharjah (2011)

5. Chen, Q, Yang, BC: A survey on the study of Hilbert-type inequalities. J. Inequal. Appl. 2015, 302 (2015)

6. Yang, BC: On Hilbert's integral inequality. J. Math. Anal. Appl. 220, 778-785 (1998)

7. Yang, BC: The Norm of Operator and Hilbert-Type Inequalities. Science Press, Beijing (2009)

8. Yang, BC, Brnetić, I, Krnić, M, Pečarić, JE: Generalization of Hilbert and Hardy-Hilbert integral inequalities. Math. Inequal. Appl. 8(2), 259-272 (2005)

9. Krnić, M, Pečarić, JE: Hilbert's inequalities and their reverses. Publ. Math. (Debr.) 67(3-4), 315-331 (2005)

10. Yang, BC, Rassias, TM: On the way of weight coefficient and research for Hilbert-type inequalities. Math. Inequal. Appl. 6(4), 625-658 (2003)

11. Yang, BC, Rassias, TM: On a Hilbert-type integral inequality in the subinterval and its operator expression. Banach J. Math. Anal. 4(2), 100-110 (2010)

12. Azar, L: On some extensions of Hardy-Hilbert's inequality and applications. J. Inequal. Appl. 2009, Article ID 546829 (2009)

13. Bényi, A, Oh, C: Best constant for certain multilinear integral operator. J. Inequal. Appl. 2006, Article ID 28582 (2006)

14. Kuang, JC, Debnath, L: On Hilbert's type inequalities on the weighted Orlicz spaces. Pac. J. Appl. Math. 1(1), 95-103 (2007) 
15. Zhong, WY: The Hilbert-type integral inequalities with a homogeneous kernel of $-\lambda$-degree. J. Inequal. Appl. 2008, Article ID 917392 (2008)

16. Hong, Y: On Hardy-Hilbert integral inequalities with some parameters. J. Inequal. Pure Appl. Math. 6(4), Article 92 (2005)

17. Zhong, WY, Yang, BC: On a multiple Hilbert-type integral inequality with the symmetric kernel. J. Inequal. Appl. 2007, Article ID 27962 (2007). doi:10.1155/2007/27962

18. Yang, BC, Krnić, M: On the norm of a multi-dimensional Hilbert-type operator. Sarajevo J. Math. 7(20), 223-243 (2011)

19. Krnić, M, Pečarić, JE, Vuković, P: On some higher-dimensional Hilbert's and Hardy-Hilbert's type integral inequalities with parameters. Math. Inequal. Appl. 11, 701-716 (2008)

20. Krnic, M, Vuković, P: On a multidimensional version of the Hilbert-type inequality. Anal. Math. 38, 291-303 (2012)

21. Rassias, MT, Yang, BC: On half-discrete Hilbert's inequality. Appl. Math. Comput. 220, 75-93 (2013)

22. Rassias, MT, Yang, BC: A multidimensional half-discrete Hilbert-type inequality and the Riemann zeta function. Appl. Math. Comput. 225, 263-277 (2013)

23. Rassias, MT, Yang, BC: On a multidimensional half-discrete Hilbert-type inequality related to the hyperbolic cotangent function. Appl. Math. Comput. 242, 800-813 (2014)

24. Rassias, MT, Yang, BC: On a multidimensional Hilbert-type integral inequality associated to the gamma function. Appl. Math. Comput. 249, 408-418 (2014)

25. Shi, YP, Yang, BC: On a multidimensional Hilbert-type inequality with parameters. J. Inequal. Appl. 2015, 371 (2015)

26. Yang, BC: An extension of a Hardy-Hilbert-type inequality. J. Guangdong Univ. Educ. 35(3), 1-7 (2015)

27. Huang, QL, Yang, BC: A more accurate Hardy-Hilbert-type inequality. J. Guangdong Univ. Educ. 35(5), 27-35 (2015)

28. Wang, AZ, Huang, QL, Yang, BC: A strengthened Mulholland-type inequality with parameters. J. Inequal. Appl. 2015, $329(2015)$

29. Yang, BC, Chen, Q: On a Hardy-Hilbert-type inequality with parameters. J. Inequal. Appl. 2015, 339 (2015)

30. Shi, YP, Yang, BC: A new Hardy-Hilbert-type inequality with multiparameters and a best possible constant factor. J. Inequal. Appl. 2015, 380 (2015)

31. Kuang, JC: Real and Functional Analysis (Continuation), vol. 2. Higher Education Press, Beijing (2015)

32. Kuang, JC: Applied Inequalities. Shandong Science Technic Press, Jinan (2004)

\section{Submit your manuscript to a SpringerOpen ${ }^{\circ}$ journal and benefit from:}

- Convenient online submission

Rigorous peer review

- Immediate publication on acceptance

- Open access: articles freely available online

- High visibility within the field

- Retaining the copyright to your article 